\title{
Masked Emotions: Do Face Mask Patterns and Colors Affect the Recognition of Emotions?
}

\author{
Olesya Blazhenkova ${ }^{1}$
}

Kivilcim Dogerlioglu-Demir²

1 Assistant Professor (corresponding author)

Sabanci University

Faculty of Arts and Social Sciences

Orta Mahalle,

Üniversite Caddesi No:27

34956 Tuzla-Istanbul, Turkey

Telephone: +902164833183

Email olesya.blazhenkova@sabanciuniv.edu

\section{Assistant Professor}

Sabanci University

Sabanci Business School

Orta Mahalle,

Üniversite Caddesi No:27

34956 Tuzla-Istanbul, Turkey

Tel:+90-216-4839677

e-mail:kivilcimdd@sabanciuniv.edu 
Masked Emotions: Do Face Mask Patterns and Colors Affect the Recognition of Emotions?

\begin{abstract}
Previous research has shown that face masks restrain the ability to perceive social information and readability of emotions. These studies mostly explored the effect of standard medical, often white masks on emotion recognition. However, in reality, many individuals prefer masks with different styles. We investigated whether the appearance of the mask (pattern-angular vs. curvy and color-black vs. white) affected the recognition of emotional states. Participants were asked to identify the emotions on faces covered by masks with different designs. The presence of masks impeded emotional recognition, dropping the accuracy and confidence and increasing reaction times. There were no main effects of angularity vs. curvature or color on emotion recognition, which suggests that mask design may not impair the recognition beyond the effect of mere mask wearing. Besides, we found relationships between individual difference variables such as mask wearing attitudes, mask design preferences, individual traits and emotional recognition. The majority of participants demonstrated positive attitudes towards mask wearing and preferred non-patterned black and white masks. Preferences for white masks were associated with better emotional recognition of masked faces. In contrast, those with negative attitudes towards masks showed lower performance in emotional recognition for masked faces, preferring patterned more than plain masks, perhaps viewing masks as a fashion item rather than a necessity. Moreover, preferences to wear patterned masks were negatively related to actual wearing masks indoors and perceived risks of COVID.
\end{abstract}




\section{Significance Statement}

In response to the COVID-19 pandemic, many people around the world wear standard, often white, medical face masks in public, covering a major part of the face. However, as pandemic continues, and mask wearing becomes the 'new normal', individuals opted for stylish face masks, thus many brands produced non-medical face masks with vibrant patterns and motifs. As face masks have become a staple in our wardrobes, more and more people use masks with a variety of designs. While previous research mostly studied the effects of standard white masks, we investigated whether the appearance of the mask (the pattern-angular vs. curvy and the color-black vs. white) affects the recognition of emotional states. Our findings suggest that these designs may not further impair readability of emotions, at least not much more than standard masks. We also found that negative attitudes towards mask wearing were negatively related to accuracy and confidence in emotional recognition of only masked but not unmasked faces. This finding is important as it suggests that those who have unfavorable mask perceptions might have reduced sensitivity to reading emotions specifically on masked faces. Our results further demonstrated that most individuals preferred black and white masks. Interestingly, those with negative attitudes towards masks and those estimated COVID-19 risks lower preferred patterned masks, perhaps viewing masks as an accessory rather than a necessity. Current study opens avenues for future studies on the effects of non-standard masks on emotional recognition.

Keywords: COVID-19, face mask; angularity vs. curvature; black vs. white; face mask perceptions 
In early 2020, COVID-19 turned into a global public health emergency, affecting the lives of millions of people. Wearing a face mask in public became a requirement in many countries (Silchenko \& Visconti, 2021) as one of the major means to prevent the transmission of the virus (Marini et al., 2021). The World Health Organization suggests wearing a mask for both preventing the spread of the COVID-19 and protection purposes especially in low air circulation places such as schools, closed buildings, etc. (WHO, 2020). More than a year after COVID-19 pandemic emerged, face mask use continues. Some even refer to the usage of face masks as the 'new normal' (Corpuz, 2021). Amid widespread recommendation to wear face masks in public, there is resistance from some people to do so. Despite the clear benefits of the practice, such a resistance stems from reasons such as inconvenience of wearing masks, perception of an infringement of independence or perceived undesirable appearance (Howard, 2020). As a result, many people opted for individualized self-made or branded stylish face masks that may have shifted the perception of masks as accessories rather than medical devices. In fact, many brands have produced non-medical face masks with vibrant patterns and motifs. As face masks make social interactions more difficult, creating social barriers was also reported to be one of the main determinants that shape negative face mask perception (Howard, 2020; Taylor \& Asmundson, 2021). Many studies showed that face masks inhibit the capability to perceive a lot of social information expressed by faces (Bani et al., 2021; Carbon, 2020; Freud et al., 2020; Marini et al., 2021).

Faces provide crucial information that allows for identifying others; it is the most distinguishing and commonly used body part to read a person's identity, age, sex, trait characteristics, intentions and emotions (Bruce \& Young, 1986; Willis \& Todorov, 2006). Individuals mostly rely on the whole face when recognizing an emotion (Baron-Cohen et al., 
1997a; Smith et al., 2005; Wegrzyn et al., 2017) and whether the focus is on the upper or lower facial areas depends on the specific emotion (Ekman et al., 1972). Therefore, it is not surprising that occlusion of salient face regions may significantly impair readability of certain emotions (Bassili, 1979; Roberson et al., 2012).

Past research has demonstrated that the eyes are the most salient part of the face. Research in face processing showed that individuals pay the utmost attention to eyes when processing face (Argyle, 1970; Blazhenkova, 2017; Goldstein \& Mackenberg, 1966; Itier et al., 2007; James et al., 2010; Janik et al., 1978). In effect, studies that employ facial stimuli that use both positive and negative contrast demonstrate that the eye region is the major factor in facial recognizability (Gilad et al., 2009). Although the contrast of the rest of the face can affect face processing, just putting the eyes in 'positive' restores the recognizability of the face (Fisher et al., 2016). Baron-Cohen et al. (1997a) created an emotional recognition test ('Reading the Mind in the Eyes Test') based on eyes area cropped out of the face that aimed to test 'theory of mind' or social sensitivity. This study examined the role of eyes in attribution of mental states (happy, sad, angry, etc.) and complex mental states (admiration, interest, thoughtfulness, etc.) and found that for the basic emotions, the whole face is more informative than either the eyes or the mouth. However, for the complex mental states, eyes were as communicative as the whole face.

Mouth region was also found to be a salient part of the face involved in expressing emotions (Beaudry et al., 2014; Blais et al., 2017; Blazhenkova, 2017; Bombari et al., 2013; Calvo et al., 2014; Eisenbarth \& Alpers, 2011; Smith et al., 2005), albeit secondary to the eye region (Blazhenkova, 2017; McKelvie, 1995; Pellicano et al., 2006). Covering the lower portion of the face was found to impede the perception of happiness since smiling - a sign of happiness is characterized by a contraction of the Zygomaticus Major muscle that extends from the 
cheekbone to the corners of the mouth (Fischer et al., 2012). When individuals were asked to focus on the upper region of a happy face (vs. the lower region of the face), detection of happiness was severely impaired as the eyes are not as relevant as the mouth for the identification of happiness (Bombari et al., 2013). Further, some eye-tracking studies reveal that the mouth area is more fixated during the recognition of happiness (Beaudry et al., 2014; Eisenbarth \& Alpers, 2011). Though most studies show that mouth area is focused when happiness is detected (Blais et al., 2012; Kotsia et al., 2008; Eisenbarth and Alpers, 2011; Fischer et al., 2012), when Duchenne smiles are observed (Okazaki et al., 2021; Sheldon et al., 2021), eyes alone are diagnostic as Duchenne refers to smiles in which both the muscle that orbits the eye and Zygomaticus Major are active.

Taken together, these studies imply that occlusion of the mouth region of the face should adversely affect identification of emotions that significantly rely on the processing of the lower part of the face such as happiness (except for faces with Duchenne smiles), sadness and anger (Fischer et al., 2012; Kret \& de Gelder, 2012), as well as surprise and disgust (Bassili, 1979). (Bassili, 1979) suggested that the bottom of the face is utilized for the recognition of happiness, surprise, and disgust, whereas the opposite is true for sadness and fear. For identification of anger, both areas are equally important. Research have argued that emotion recognition from the face is actually rather complex. People process emotions in either a featural or a configural manner or both. Whereas features refer to certain face parts, such as the eyes or mouth, configurations refer to relational information such as interrelationships between the mouth and the eyes. That is, certain emotions are not derived by looking at one region (Bombari et al., 2013; McKelvie, 1995; Prkachin, 2003). In a study, where participants viewed intact, blurred or scrambled faces with different types of information (featural, configural, or both), 
happiness was found to be recognized more easily and rapidly than the other emotions irrespective of the conditions. This suggests that happiness is mainly recognized in a featural manner, by looking at the smiling mouth. Happiness recognition should therefore be significantly affected by covering the mouth unless there is a Duchenne smile which includes smiling of the eyes. Fear, too, was found to be recognized in a featural manner. Though fear is mainly identified by the big open eyes, the mouth is also instrumental in detecting fear (Bombari et al., 2013). Hence, occluding the mouth, fear detection could be impaired, yet to a lesser degree compared to happiness. Sadness is recognized in a configural way (Bombari et al., 2013), eliciting longer fixations on the centre of the face (Bombari et al., 2013) or the eye area (Bassili, 1979; Beaudry et al., 2014; Eisenbarth \& Alpers, 2011; Wegrzyn et al., 2017). At the same time, eyebrows and mouth are used for the recognition of sadness (Calvo et al., 2014; Smith et al., 2005). Sadness recognition, then, might be affected by occlusion of the mouth, albeit to a lesser degree compared to happiness. Anger is identified both in a featural and configural way. Mouth is not necessarily a relevant feature for the recognition of anger (Bombari et al., 2013) as it involves contraction of the Corrugator Supercilii muscle as a result of furrowing of the brow (Fischer et al., 2012). Anger should be less impaired by covering the mouth as the upper part of the face is focused when detecting anger. Past researchers often excluded surprise and disgust in their emotion recognition studies because those two are confused with anger and fear, respectively (Bombari et al., 2013; McKelvie, 1995; Prkachin, 2003). Yet, the mouth area was suggested to be the most useful for the recognition of surprise and both the nose and mouth area are used for the recognition of disgust (Calvo et al., 2014; Smith et al., 2005) so one could expect impairment of such emotions as a result of occlusion of the mouth. 
Research on the effect of occlusion of particular face regions on emotion recognition accuracy is especially relevant in the context of understanding the effects of face masks under the ongoing COVID-19 pandemic. By covering about $60 \%$ of the face that is pertinent to emotional expression (Carbon, 2020; Freud et al., 2020), face masks interfere with the recognition of its wearer's emotional state (Freud et al., 2020; Marini et al., 2021), resulting in misinterpretation of emotions (Carbon, 2020). Carbon showed that applying a mask on faces resulted in a significant accuracy decline in reading basic emotions such as happiness, anger, disgust and sadness except for fear and neutral. For confidence ratings, along with others, fear and neutral emotions also revealed significant drops. Further analyses demonstrated some nonrandom patterns of confusions between the emotional states in unmasked faces, which became more pronounced for the masked faces. For example, disgusted faces were misinterpreted as angry in almost $40 \%$ of the cases ( $2 \%$ of the cases in the no mask condition). In addition, Carbon found that different emotional states were more frequently confused with a neutral state in the masked condition. A similar study, this time with medical students, showed that students made more errors when faces were presented with face masks for emotions of happiness, anger, sadness but not for fear (Bani et al., 2021). Yet, in another study (Ramachandra \& Longacre, 2022), half of the participants were shown pictures of the whole face of a woman and the other half was shown only the eyes of the same woman expressing the same emotions (similar to a mask covering the mouth). The results revealed a significant difference in emotion recognition between facial vs. eyes conditions for all emotions except sadness and distress. While the recognition accuracy was the best for happiness in the face condition, it was the best for surprise in the eyes condition, and it was the worst for distress in both face and eyes conditions. Surprise and sadness were better in the eyes-only condition. Other studies demonstrated that face masks 
impact recognition of expressions significantly involving lower face features the most (disgust, anger), and emotions involving upper face features the least (fear, surprise) (McCrackin et al., 2021). In sum, previous evidence on the role of face masks in emotion recognition suggests that, overall, masks impede emotional recognition, however, this effect for specific emotions varies from study to study. Fear seems to be the least affected emotion and all other emotions seem to be adversely influenced by occlusion of the mouth, yet there seems to be no consensus as to how other emotions are affected. One reason for inconsistencies between studies could be the use of different stimuli in experiments across papers. Carbon (2020), for instance, showed that recognizing emotions of elderly faces was much harder than that for middle-aged or young faces. Hence, age differences among the models used in these studies might be one factor. Also, using different models with different ability to express emotions may play a role. For example, Carbon used a professional data set with models who can clearly pose emotional states and found relatively high accuracy in recognition of emotions in masked faces. Moreover, sample characteristics might have contributed to the observed inconsistencies in the literature. An examination of eye movements showed that while Western individuals' eye fixations were scattered evenly across the faces, Eastern individuals mainly focused on the eyes (Jack et al., 2009). In addition, there is research that demonstrates that as individuals' exposure to masks increases, they actually get better at reading emotions (Barrick et al., 2020). Hence, timing of execution of these studies may matter. Those which are closer to the beginning of the pandemic may demonstrate lower accuracy ratings compared to more recent ones.

Overall, previous research indicates that emotional states are harder to decipher and easily confounded when a target occludes the mouth region by wearing a face mask. It must be noted, however, that the above-mentioned studies focused on mask (medical) vs. no mask 
conditions generally applying standard often white medical masks. However, in reality, individuals use different types of face coverage with different appearance (e.g., patterned and colored masks). There are only a few studies that investigated the effect of non-medical and nonwhite masks on emotion recognition. Employing bi-state electrochromic displays, Genç et al. (2020) created smart masks -- a Mouthy Mask (reproducing the image of the mask wearer's mouth) and a Smiley Mask (using an emoji instead of a representation of a mouth). Results showed that individuals on average preferred visualizations representing the wearer's mouth as a means to mitigate facial expression occlusion (Genç et al., 2020). Though specific emotions were not studied in this research, overall perceived understandability of the emotions significantly improved when smart face masks were used. Further, Marini et al. (2021) demonstrated that in contrast to standard medical face masks, transparent masks significantly increase the capability to recognize emotional expressions (Marini et al., 2021) for all emotions (happiness, sadness, fear) except for neutral. In fact, no significant differences were observed in accuracy between non-mask and transparent mask conditions across all emotions.

\section{Emotion recognition: effects of mask patterns and color}

In the current study, we aimed to examine factors related to mask appearance that might play a role in recognition of emotional states. We contend that the pattern (angular vs. curvy patterns) or the color (black vs. white) of the mask may serve as an additional input when reading emotions. To our knowledge, no researcher to date has studied the effect of masks with different patterns or color and how they affect emotional recognition.

Our motivation to study the effects of angularity vs. curvature is based on the research in multiple disciplines, from art and aesthetics to neurobiology, from visual cognition to social psychology and marketing. These works highlighted differences in perception of angular vs. 
curved shapes (Carbon, 2010; Hussain, 1972; Palumbo et al., 2015). The curved over sharp preference was claimed to be a basic visual primitive evolutionary-based function (Carbon, 2010). A cross-cultural study demonstrated that curvature is one of the dimensions that drives preference of people from different cultures around the world (Gómez-Puerto et al., 2018). Angular stimuli were found to be associated with threat (Aronoff et al., 1988; Aronoff et al., 1992) and aggressive emotions (Hevner, 1935; Lundholm, 1921; Poffenberger \& Barrows, 1924). Furthermore, Bar and Neta suggested that curvature preference could be due to threat avoidance (Bar \& Neta, 2006). Palumbo et al. found that angular polygons were associated with negative emotions, whereas curved polygons were associated with positive emotions (Palumbo et al., 2015). Blazhenkova and Kumar also found associations between abstract angular shapes with negative emotions and curved shapes with positive emotions (Blazhenkova \& Kumar, 2018). Different attributions for angularity vs. curvature in terms of emotional valence and arousal (e.g., unpleasant, agitating, and harsh vs. pleasant, gentle, quiet) were also reported in earlier research on aesthetic perception (Lundholm, 1921; Poffenberger \& Barrows, 1924). Besides, angularity vs. curvature convey different meanings and benefits in marketing literature. For instance, while angular brand logos denote conflict and aggressiveness, round logos are seen as harmonious and gentle (Jiang et al., 2015; Zhang et al., 2006). As angularity depicts hardness and aggressiveness, a juice coming in an angular package (compared to a curvy one) leads consumers to experience the product taste as more intense (Becker et al., 2011). Blazhenkova and Dogerlioglu-Demir (2020) investigated the effects of pills' shape (angular vs. curvy) on the perceived efficacy of the medicine, bodily sensations and emotions and found that the angular pills trigger more activations in the body compared to curvy pills. While angularity was found to be linked with an energizing effect, roundness was associated with a calming effect. Further, 
angularity was linked with negative emotions such as anxiety, fear, anger, and irritation. However, a positive relief emotion was evoked by curved shapes (Blazhenkova \& DogerliogluDemir, 2020). Literature also suggested that curved shapes resemble facial emotions expressing happiness (curves in cheeks, smiling mouth) whereas angular shapes resemble angry expressions (v-shaped angles in the eye-brows) (Bassili, 1979; Aronoff, Woike, \& Hyman, 1992). Infants' rounded head and facial features were associated with warmth and protectiveness (Papanek, 1995), while sharp or angular shapes such as sharp edges of the teeth of a tiger are associated with danger. These results therefore suggest that the effect of angularity vs. curvature is a robust finding and it may be related to face perception. Thus, we expected to find that masks with angular patterns may enhance the perception of anger (and other negative emotions) whereas masks with curved patterns may enhance the perception of happiness (and other positive emotions).

Another characteristic of visual appearance related to emotional processing is color, and we also expected to see its effect on recognition of emotions on masked faces. Past research tested association between colors and emotions and found that whereas white is seen as a happy color (Clarke \& Costall, 2008), black is perceived as a sad color (Cimbalo et al., 1978). Similarly, white is associated with positivity and joy and black is associated with negativity, depression, evil and even death (Sliburyte \& Skeryte, 2014). White is generally linked to sincerity as it is associated with characteristics such as cleanness, simplicity, and peace. Black also stands for sophistication and glamour and power (Labrecque \& Milne, 2012). In the fashion world, black is the most worn color for dressy occasions (Funk \& Nelson, 2006). Black expresses status and elegance (e.g., black tie events, little black dresses and tuxedos) (Labrecque \& Milne, 2012). Although these associations have been observed across cultures (for instance, 
black is consistently associated with 'expensive' and 'powerful' across cultures, (De Bortoli \& Maroto, 2001), culture specific linkages have also been demonstrated (Hanada, 2018). Additional meaning associations of "formal" (Brazil, Colombia, PRC, and Taiwan) and "masculine" (Austria, Hong Kong, the United States) were evident in some countries (Madden et al., 2000). In all countries, white is associated with "peaceful," "gentle," and "calming." Some cultures associate "beautiful" (Brazil, Hong Kong, PRC, United States) with white (Madden et al., 2000). White is associated with cleanliness and purity as well as emptiness (Saito, 1996) and emotionlessness (Hanada, 2018) in Asian cultures and mourning particularly in China (De Bortoli \& Maroto, 2001). Based on this literature, in our study, we anticipated to observe that black masks (and darker patterned masks) enhance the perception of negative emotions whereas white masks (and lighter patterned masks) may enhance the perception of positive emotions in masked faces.

Furthermore, we expected that individual difference variables (such as emotional processing traits and attitudes towards masks) may play a role in emotional recognition in masked faces. Previous research has indicated that attitudes towards masks may influence emotion recognition. In a recent study by Bierman et al. (2021), a stronger negative bias was found in happiness and trustworthiness judgments of faces with masks for those who attribute less protective effect to masks, have a lower experienced risk concerning COVID-19 and those who see face mask wearing as a burden. Further, for trustworthiness appraisals, the negative bias was stronger for those who tend to comply less with the face mask wearing rules (Biermann et al., 2021). Further, face masks by themselves may also have negative associations with danger (e.g., pollution, disease) and covering face even by a non-mask fabric (e.g., scarf) may trigger the feelings of insecurity (i.e., someone is hiding true emotions or having bad and unclear 
intentions). There is also evidence showing that certain face coverings (e.g., niqab) are associated with an out-group and impact readability of emotions of women wearing niqab (Fischer et al., 2012). Though some emotional signals may still be perceived, observers may be biased toward seeing more negative emotions, when they are guessing about what is going on beneath the veil. No studies to date, however, examined the effect of mask appearance on the attitudes towards masks. Attitudes towards these different masks (patterned or different colors) may relate to emotional recognition, and we aimed to explore this.

Finally, we examined individual differences in emotional processing (i.e., richness of emotional experiences or anxiety) in relation to perception of emotions on masked faces. Previous literature suggested that individual differences may predict emotional recognition in general, and also in faces when only eyes are present. For example, persons with higher overall social competence were found to be better at identifying unmasked expressions, while persons with lower trait extraversion and higher trait agreeableness were better at recognizing masked expressions (McCrackin et al., 2021). Besides, people with difficulties in emotional processing such as those with high functioning autism or Asperger syndrome were found to have impaired emotion recognition from the eyes alone more than other populations, Wheelwright, \& Jolliffe , 1997b). More recent studies showed that higher degree of autistic traits predicted a greater difficulty in recognizing emotions both with and without masks (Pazhoohi et al., 2021; Ramachandra \& Longacre, 2022). Anxiety was also found to be related to altered emotional recognition, so that individuals with high-trait anxiety were shown to identify fear significantly better. However, other emotions did not differ across low-trait anxiety and high-trait anxiety groups (Surcinelli et al, 2006). Other reports showed that anxious individuals tend to have a negative bias and to identify neutral faces as angry (Deighton, R. M., \& Traue, 2007; Demenescu 
et al., 2010). No studies examined anxiety in relation to emotional recognition of masked faces. As the mere presence of masks may trigger anxiety (Saint et al., 2021) due to the ambiguity the mask creates (people hiding face and possibly intentions), we expected even more negative bias in anxious individuals reading emotions from masked faces.

Our current research aimed to investigate the effect of individual difference variables on emotion recognition and attitudes towards masks when masks of different appearance are used. Consistent with previous studies, we expected to find that people with richer emotional experiences would have better recognition of emotions on masked faces, and people with emotional difficulties such as anxiety may have impaired emotional recognition in masked faces. Moreover, we aimed to further explore whether individual differences in emotional processing relate to emotional recognition depending on mask appearance.

At first blush, because the recognition of basic emotions is better for the whole face than the parts, consistent with other studies, it may be predicted that wearing any face mask might interfere with accurate emotion evaluations. We further suggest that the patterns on the mask as well as its color may serve as an additional factor when judging emotions. The present research aims to fill this knowledge gap by investigating recognition of emotions as well as potential confusion of emotional states due to wearing patterned or colored face masks. To our knowledge, our research constitutes the first step toward understanding how visual appearance of occluders may interfere with emotional processing of faces.

Hypothesis 1 (Mask). Emotional states on masked faces are less recognizable resulting in lower emotional recognition accuracy, longer response times and lower confidence levels. We do not have specific predictions about the effects of specific patterns or colors on the overall emotional recognition but expect possible differences. 
Hypothesis 2 (Mask*Emotion). The presence of the mask and its design (curvy vs. angular and black vs. white) impact the recognition of the specific emotional states. For the masked faces, the recognition of emotions expressed with a significant involvement of the lower part of the face is more impaired than for other emotions. We also expect that angular and predominantly black designs would result in more negative emotion perceptions whereas curvy and predominantly white patterns would yield more positive emotion perceptions.

Hypothesis 3 (Individual differences). Individual differences in emotional processing and attitudes towards masks are related to recognition of emotions in faces with masks with different designs. Emotional recognition in people with higher anxiety and negative attitudes towards mask wearing is more impaired by masks. We do not have specific predictions about the relationship between individual differences and recognition of emotional states in faces with specific patterns on masks but expect possible differences.

\section{Method}

\section{Participants}

Participants were students from a Turkish University studying in the English language. They were recruited via University Sona systems and received bonus course credits for their participation. Initially, 157 students participated in the study. Among them, 10 failed the attention check question ('Now please ignore this question and mark 'agree'), 7 did not disagree with another attention check statement ('I am not a human') and therefore were excluded from the analysis. In addition, we excluded 5 participants who took too long to respond (time to submit the page exceeded $2 S D^{1}$ ). Eventually, the data from 137 participants (91 females, 18-29

\footnotetext{
${ }^{1}$ The outliers were removed since the response time data was used in the analysis.
} 
years old, mean age 22 years old) data were retained for the analysis. The study was conducted in May 2021, after more than a year since the beginning of the pandemic and fully remote learning (the campus was closed).

\section{Procedure}

Participants completed Masks' rating task as well as a few individual differences selfreport measures assessing attitudes towards masks and emotional processing. They provided informed consent and completed the study online via Qualtrics software.

\section{Materials}

\section{Masks' rating task}

Face images were taken from MPI FACES database (Ebner et al., 2010), the same as used by Carbon (2020). We only included 1 young male and 1 young female faces. Masks on faces were photoshopped (Figure 1) using pictures with angular and curved patterns obtained from istockphoto. Masks' rating task consisted of 168 Masked Faces trials: (Sex - 2 [male, female], Emotions - 6 [afraid, angry, disgust, happy, neutral, sad], Patterns - 7 [3 angular, 3 curved, plain], Color - 2 [predominantly white, predominantly black - we included purely black and white masks, and for the patterned masks we applied inversion]), 14 Masks Only trials (Patterns - 7, Color - 2), and 12 Unmasked Faces trials (Sex - 2, Emotions - 6). Masked Faces trials were administered first, followed by Masks Only trials, and finally by Unmasked Faces trials (faces without masks were administered last to prevent the influence of unmasked faces on recognition of masked faces). Each participant completed all the trials (the order of trials was randomized within each trial type). For each trial, a stimulus and the respective questions were presented on one separate page. The response time was not restricted but recorded, and participants proceeded to the next trial by clicking the 'next' button. 


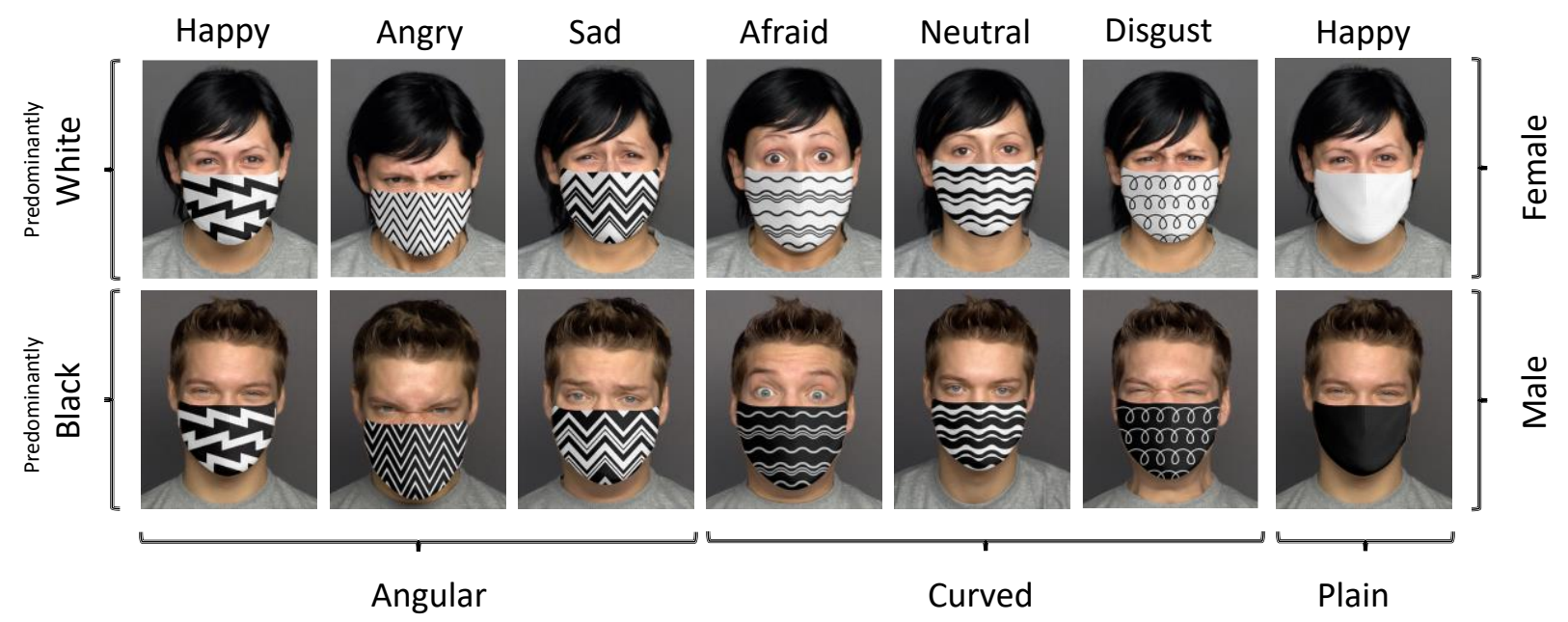

Figure1. Examples of stimuli used in the Masks' rating task.

In each of the Masked Faces or Unmasked Faces trials, participants viewed a masked face and had to indicate a person's emotional state (choose from a list of 7 emotions: afraid, angry, disgust, happy, neutral, sad, and surprise). Next, they had to indicate their confidence in assessing emotion from 1 (very unconfident) to 7 (very confident). Both accuracy and response time (time to first click, time to page submit) were recorded.

In Masks Only trials, participants were asked to 'indicate the extent the pattern on the above mask is...' 1) on the scale from 1 (Extremely Curvy) to 7 (Extremely Angular), 2) on the scale from 1 (Extremely Black) to 7 (Extremely White). These questions were included to check the shape and color manipulations. Next, participants rated their preferences: 'Imagine that you have to attend a social event indoors, and there is a rule to wear a mask. How likely is it that you would wear a mask with this pattern?' on the scale from 1 (Not likely at all) to 7 (Very much likely). 


\section{Range and differentiation of emotional experience scale (RDEES)}

This self-report assesses individual differences in emotional complexity defined as having emotional experiences that are broad in range and well differentiated (Kang \& Shaver, 2004). On a 5-point Likert scale, participants rated their agreement with 14 statements from the 2 subscales: Emotional Differentiation (e.g., 'I am aware of the different tones or subtleties of my various emotions'; $\alpha=.79$ ) and Emotional Range (e.g., 'I experience a wide range of emotions'; $\alpha=.82)$.

\section{Short State-Trait Anxiety Inventory (SSTAI)}

The abbreviated version of Spielberger State-Trait Anxiety Inventory (STAI; Spielberger et al., 1970) assesses individual anxiety levels and includes 5-items per State (STAIS-5; $a=.91$ ) and Trait Anxiety (STAIT-5; $\alpha=.86$ ) scales (Zsido et al., 2020).

\section{Face Mask Perceptions Scale (FMPS)}

FMPS (Howard, 2020) is 32-item questionnaire that assesses negative attitudes towards mask wearing on 8-dimensions: Comfort (e.g., 'Face masks disrupt my breathing”; $\alpha=.91$ ), Efficacy Doubts (e.g., 'Face masks provide few health benefits'; $\alpha=.86$ ), Access (e.g., 'Face masks are too expensive'; $\alpha=.86$ ), Compensation (e.g., 'I already social distance'; $\alpha=.86$ ), Inconvenience (e.g., 'It is hard to develop the habit of wearing a face mask'; $\alpha=.83$ ), Appearance (e.g., 'Face masks look silly'; $\alpha=.97)$, Attention (e.g., 'Face masks make people seem untrustworthy'; $\alpha=.94$ ), Independence (e.g., 'I do not like feeling forced to do something'; $\alpha=.89)$. In addition, we developed 5 new questions for Emotion scale, assessing difficulty in reading emotions (e.g., 'I can't understand people's emotions clearly when they wear face masks'; 'I can't express my emotions clearly when I wear face masks'; 'I do not feel emotionally connected with people who wear face masks'; 'I think people cannot clearly understand my 
emotions when I wear face masks'; 'People cannot feel emotionally connected with me when I wear face masks'; $\alpha=.92$ based on the data of the current study). Besides, we included unvalidated 4-items scale, Face Mask Perceptions, FMP+, (e.g., 'People should wear face masks in public'), assessing positive attitudes towards masks not only in the context of COVID but also in general (Howard, 2020).

\section{Mask wearing habits and COVID-19 risks estimation}

Participants responded to 'Within the six months, how often have you worn a face mask outdoors when going into public?' and 'Within the six months, how often have you worn a face mask indoors when going into public?' on the scale from 1 (Never) to 7 (Every time). They also responded to 'Please indicate the current situation with COVID-19 in the country where you reside now. How would you estimate the risk with COVID-19 in your country?' on the scale from 1 (Very risky) to 7 (Not risky at all).

\section{Results}

\section{Emotion Recognition Accuracy}

$6 \times 5$ Repeated Measures ANOVA was conducted to assess the impact of two withinsubjects variables, Emotion (Happy, Angry, Sad, Afraid, Neutral, Disgust) and Mask (White, Black, Angular, Curved, Unmasked) on participants' emotion recognition accuracy ${ }^{2}$.

The main effect of Emotion was statistically significant, $F(3.279,445.953)=435.906, p<$ $.001\left(\eta_{\mathrm{p}}^{2}=.762\right)$. Happy $(M=.978, S D=.006)$, Angry $(M=.979, S D=.005)$, and Neutral $(M=$ $.954, S D=.009$ ) emotions' recognition accuracy was very high. While there were no differences in recognition accuracy for these emotions, Happy was recognized significantly better than all

\footnotetext{
2 The adjustments for multiple comparisons were done with Bonferroni correction. A Greenhouse-Geisser adjustment of the degrees of freedom was performed due to a sphericity assumption violation.
} 
other emotions (all ps $<.001$ ), whereas Angry and Neutral were recognized significantly better than $\operatorname{Sad}(M=.709, S D=.023)$, Afraid $(M=.224, S D=.020)$, and Disgust $(M=.749, S D=$ .018) (all ps <.001). Afraid had the lowest recognition accuracy compared to all emotions (all ps<.001).

The main effect of Mask was statistically significant, $F(2.603,353.993)=96.609, p<$ $.001\left(\eta_{\mathrm{p}}^{2}=.415\right)$. Unmasked faces were recognized significantly better than all masked faces (all $p s<.001)$, and there were no other significant differences between recognition rates for faces covered with masks of different patterns and colors.

The interaction between Emotion and Mask was also significant, $F(8.636,1174.491)=$ 29.302, $p<.001\left(\eta_{\mathrm{p}}{ }^{2}=.177\right)$. Figure 2 A shows recognition accuracy for different emotions and mask's patterns. Unmasked faces were recognized considerably better for Afraid and Disgust emotions. Pairwise comparisons demonstrated the following significant differences: for Happy emotion - superior recognition of unmasked compared to all other masked conditions (all ps < .045); for Angry emotion - superior recognition of unmasked compared to curved masked condition $(p=.043)$; for Sad emotion - superior recognition of curved masked compared to angular masked condition $(p=.034)$; for Afraid emotion - superior recognition of unmasked compared to all other masked conditions (all $p s<.001$ ); for Disgust emotion - superior recognition of unmasked compared to all other masked conditions (all $p s<.001$ ), superior recognition of white masked compared to angular masked condition $(p=.028)$ as well as curved masked condition $(p=.022)$. 

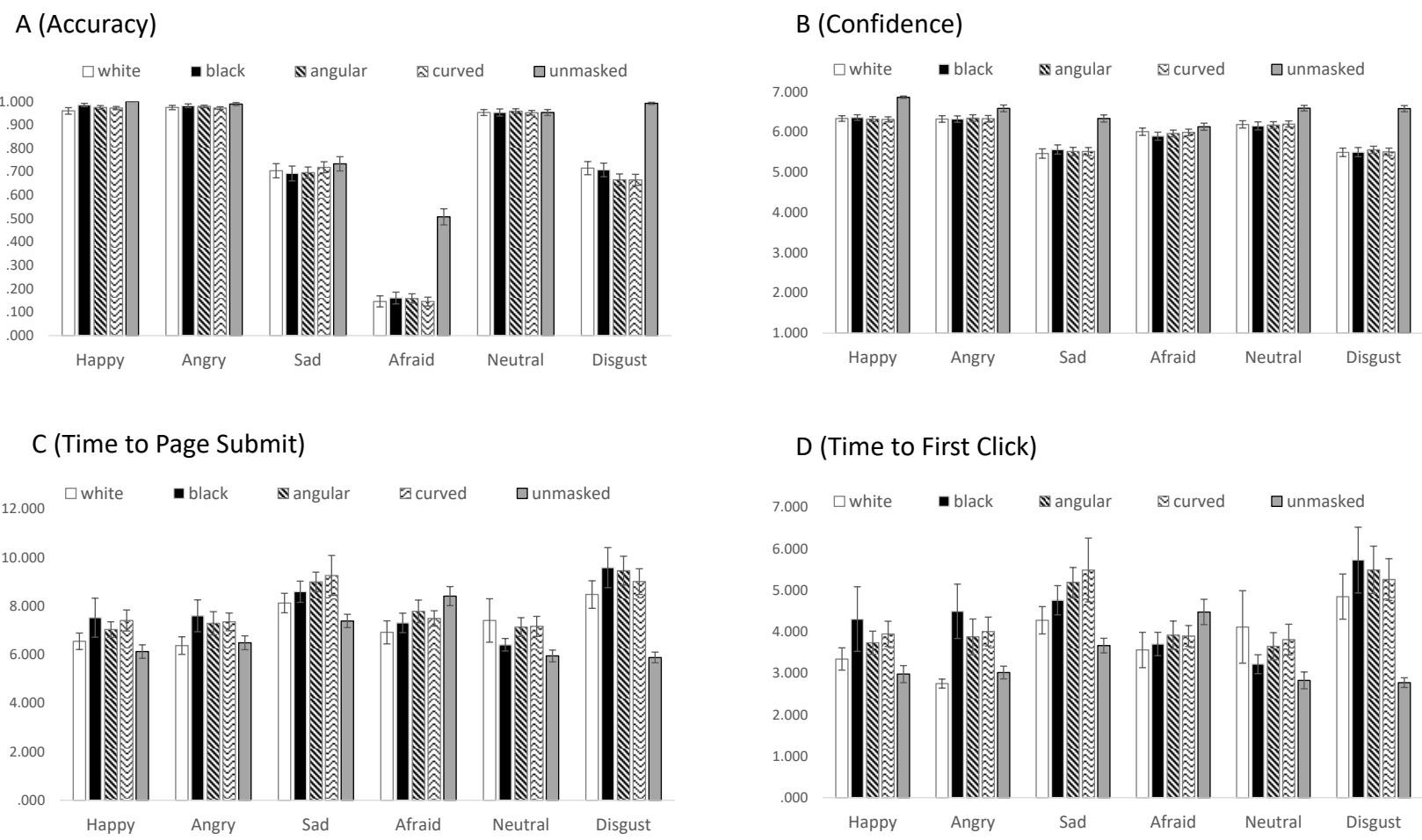

Figure 2. Recognition accuracy, Confidence, and Response time for Masks' rating task.

\section{Emotion Recognition Confidence}

The same as for accuracy analyses, $6 \times 5$ RM ANOVA assessed the effect of Emotion and Mask on confidence in accuracy of emotion recognition.

The main effect of Emotion was statistically significant, $F(3.925,533.789)=39.930, p<$ $.001\left(\eta_{\mathrm{p}}{ }^{2}=.227\right)$. Confidence for Happy $(M=6.440, S D=.051)$ was higher than for all other emotions $[p<.058$ for Neutral, $M=6.263, S D=.076$, all other $p s<.001]$ except for Angry $(M=$ $6.384, S D=.075)$. Confidence for Angry was higher than for $\operatorname{Sad}(M=5.682, S D=.094)$, Afraid $(M=6.000, S D=.078)$, and Disgust $(M=5.730, S D=.082)$, all $p s<.001$. Confidence for Afraid was higher than for Sad and Disgust $(p s \leq .007)$ but lower than for Happy, Angry, and Neutral ( $p s \leq .005)$. Confidence for Neutral was higher than for Sad, Neutral, and Disgust ( $p s \leq$ $.005)$ but lower than for Happy and Angry $(p s \leq .005)$. Confidence for Disgust was lower than 
for all other emotions (all other $p s \leq .001$ ) except for Sad. Confidence for Sad was lower than for all other emotions (all other $p s \leq .001$ ) except for Disgust.

The main effect of Mask was statistically significant, $F(1.943,264.243)=107.069, p<$ $.001\left(\eta_{\mathrm{p}}{ }^{2}=.440\right)$. Unmasked faces were recognized with significantly more confidence than all masked faces (all $p s<.001$ ), and there were no other significant differences.

The interaction between Emotion and Mask was also significant, $F(10.540,1433.442)=$ 13.613, $p<.001\left(\eta_{\mathrm{p}}{ }^{2}=.091\right)$. Figure 2B shows confidence in recognition for different emotions and mask's patterns. Pairwise comparisons demonstrated the following significant differences: for Happy, Sad, Angry, Neutral and Disgust emotions - superior confidence in recognition of unmasked compared to all other masked conditions (all $p s<.001$ ); for Afraid emotion - superior confidence in recognition of unmasked compared to Black and Agular (all $p s<.001$ ).

\section{Emotion Recognition Response Time (Time to Page Submit)}

The same $6 \times 5$ RM ANOVA assessed the effect of Emotion and Mask on Response Time (Time to Page Submit).

The main effect of Emotion was statistically significant, $F(449.924,607.572)=14.649$, $p<.001\left(\eta_{\mathrm{p}}^{2}=.097\right)$. RT for Happy $(M=6.926, S E=.270)$ as well as Angry $(M=7.018, S E=$ $.250)$ for were significantly lower than for $\operatorname{Sad}(M=8.470, S E=.279)$ and Disgust $(M=8.480$, $S E=.330)$, all $p s<.001 . \mathrm{RT}$ for Sad was longer than for Happy, Angry, Afraid $(M=7.579, S E=$ $.243)$ and Neutral $(M=6.809, S E=.272), p s \leq .019$. RT for Afraid was shorter than for Sad, $p=$ .019. RT for Neutral was shorter than for Sad and Disgust, $p s<.001$. RT for Disgust was longer that for other emotions $(p s<.001)$ expect for Sad and Afraid. 
The main effect of Mask was statistically significant, $F(3.631,493.868)=6.444, p<.001$ $\left(\eta_{\mathrm{p}}^{2}=.045\right)$. Unmasked faces were recognized with significantly faster than all masked faces (all $p s \leq .007)$ except for White.

The interaction between Emotion and Mask was also significant, $F(11.170,1519.129)=$ 2.550, $p<.001\left(\eta_{\mathrm{p}}^{2}=.018\right)$. Figure $2 \mathrm{C}$ shows recognition time for different emotions and mask's patterns. Pairwise comparisons demonstrated the following significant differences: for Happy emotion - faster recognition of unmasked compared to Angular and Curved masked conditions (all $p s \leq .020$ ); for Angry emotion - faster recognition of unmasked compared to curved masked condition $(p=.032)$; for Sad emotion - superior recognition of curved masked compared to Black, Angular and Curved masked conditions ( $p s \leq .030)$; for Afraid emotion - longer recognition of unmasked compared to white masked condition (all $p=.011$ ); for Neural emotion - faster recognition of curved masked compared to Angular and Curved masked conditions ( $p s \leq$ .008); for Disgust emotion - faster recognition of unmasked compared to all other masked conditions (all ps<.001).

\section{Emotion Recognition Response Time (Time to Page to First Click)}

The same $6 \times 5$ RM ANOVA assessed the effect of Emotion and Mask on Response Time (Time to First Click).

The main effect of Emotion was statistically significant, $F(4.467,607.553)=9.833, p<$ $.001\left(\eta_{\mathrm{p}}^{2}=.067\right)$. RT for Happy $(M=3.660, S E=.222)$ as well as Angry $(M=3.627, S E=.213)$ were significantly lower than for $\operatorname{Sad}(M=4.674, S E=.239)$ and Disgust $(M=4.818, S E=$ .296), all $p s<.001$. RT for Sad was longer than for Happy, Angry, Afraid $(M=3.910, S E=$ $.199)$ and Neutral $(M=3.522, S E=.228), p s \leq .019$. RT for Afraid was shorter than for Sad and 
Disgust, $p \mathrm{~s} \leq .023$. RT for Neutral was shorter than for Sad and Disgust, $p s \leq .001$. RT for Disgust was longer that for other emotions $(p s \leq .023)$ expect for Sad.

The main effect of Mask was statistically significant, $F(3.629,493.532)=6.597, p<.001$ $\left(\eta_{\mathrm{p}}^{2}=.067\right)$. Unmasked faces were recognized with significantly faster than all masked faces (all $p s \leq .002$ ) except for White.

The interaction between Emotion and Mask was also significant, $F(9.523,1295.108)=$ $2.090, p=.025\left(\eta_{\mathrm{p}}^{2}=.015\right)$. Figure 2D shows recognition time for different emotions and mask's patterns. Pairwise comparisons demonstrated the following significant differences: for Happy emotion - superior recognition of unmasked compared to Angular and Curved masked conditions ( $p s \leq .023)$; for Angry emotion - faster recognition of unmasked faces compared to Black, Angular and Curved masked conditions $(p s \leq .048)$ as well as faster recognition of White masked faces compared to Black, Angular and Curved masked faces ( $p s \leq .009)$. For Sad emotion - faster recognition of curved masked compared to Black, Angular and Curved masked conditions $(p s \leq .022)$ as well as faster recognition of White masked faces compared to Angular masked faces $(p=.039)$; for Afraid emotion - no significant differences in response time; for Neural emotion - faster recognition of curved masked compared to Angular and Curved masked conditions ( $p s \leq .029$ ); for Disgust emotion - faster recognition of unmasked compared to all other masked conditions (all $p s<.001$ ).

\section{Confusion between different emotional states}

Similar to Carbon (2020), we examined the specific confusions of different emotional states in unmasked and all masked conditions (see confusion matrix in Table 2). Overall, most of the emotional states posed by unmasked faces were not misinterpreted. One exception was Sad that was mostly confused with Afraid (18.61\%) and less so with Disgust (8.03\%); this is 
somewhat similar to Carbons' findings (Sad was mainly confused with Disgust, 20.3\%). The second exception was Afraid that was misinterpreted as Surprise in almost half of the cases (45.99\%). Afraid-Sad confusion is a common finding in the literature (Bassili, 1979). Note, we added the Surprise answer option while we did not actually have a Surprise-posing face. We did not find Angry misinterpretation as Disgusted, reported by Carbon, or any other considerable confusions for the unmasked faces.

For all kinds of masked faces, we observed very similar patterns of misinterpretations . Similar to Carbon, we found that for masked faces the characteristic confusions became more outstanding. Sad, overall, was more frequently confused with both Disgust and Afraid, but at a comparable rate (10-13\% each). Afraid became drastically confused with Surprise (82-84\%). Similar to Carbon, we found that Disgust became confused with Angry (12-22\%), though it was less pronounced than in Carbon's study (37.8\%). Unlike Carbon, we did not find that masked faces were more frequently misinterpreted as Neutral in the masked condition. Also, inconsistently with Carbon, Happy and Angry emotional states were rarely confused with others in either masked or unmasked conditions. 
Table 2. Confusion Matrix of Emotions.

\begin{tabular}{|c|c|c|c|c|c|c|c|}
\hline \multirow{2}{*}{$\begin{array}{l}\text { Expressed Emotion } \\
\text { No Mask }\end{array}$} & \multicolumn{7}{|c|}{ Perceived Emotion } \\
\hline & Happy & Angry & Sad & Afraid & Neutral & Disgust & Surprise \\
\hline Happy & 100.00 & 0.00 & 0.00 & 0.00 & 0.00 & 0.00 & 0.00 \\
\hline Angry & 0.00 & 98.91 & 0.36 & 0.36 & 0.00 & 0.36 & 0.00 \\
\hline Sad & 0.00 & 0.00 & 73.36 & 18.61 & 0.00 & 8.03 & 0.00 \\
\hline Afraid & 0.00 & 0.00 & 0.36 & 50.73 & 0.00 & 2.92 & 45.99 \\
\hline Neutral & 0.73 & 0.73 & 3.28 & 0.00 & 95.26 & 0.00 & 0.00 \\
\hline Disgust & 0.00 & 0.00 & 0.73 & 0.00 & 0.00 & 99.27 & 0.00 \\
\hline \multicolumn{8}{|l|}{ White Mask } \\
\hline Happy & 95.99 & 0.73 & 0.73 & 0.00 & 0.73 & 1.09 & 0.73 \\
\hline Angry & 0.00 & 97.45 & 0.73 & 0.36 & 0.00 & 1.46 & 0.00 \\
\hline Sad & 0.36 & 0.73 & 70.44 & 10.58 & 2.55 & 12.04 & 3.28 \\
\hline Afraid & 1.09 & 0.00 & 0.00 & 14.60 & 0.00 & 0.36 & 83.94 \\
\hline Neutral & 0.00 & 1.09 & 1.09 & 1.82 & 95.26 & 0.36 & 0.36 \\
\hline Disgust & 5.11 & 19.71 & 1.09 & 2.19 & 0.00 & 71.53 & 0.36 \\
\hline \multicolumn{8}{|l|}{ Black Mask } \\
\hline Happy & 98.54 & 0.36 & 0.36 & 0.00 & 0.00 & 0.36 & 0.36 \\
\hline Angry & 0.00 & 98.18 & 0.00 & 0.00 & 0.00 & 1.82 & 0.00 \\
\hline $\mathrm{Sad}$ & 0.36 & 0.36 & 69.34 & 12.04 & 1.09 & 13.14 & 3.65 \\
\hline Afraid & 0.00 & 0.36 & 0.73 & 16.06 & 0.36 & 0.00 & 82.48 \\
\hline Neutral & 0.00 & 0.73 & 1.82 & 0.36 & 95.26 & 0.73 & 1.09 \\
\hline Disgust & 5.47 & 18.25 & 1.82 & 3.28 & 0.36 & 70.80 & 0.00 \\
\hline \multicolumn{8}{|l|}{$\begin{array}{l}\text { Curved-patterned } \\
\text { Mask }\end{array}$} \\
\hline Happy & 97.20 & 0.43 & 0.55 & 0.18 & 0.30 & 0.85 & 0.49 \\
\hline Angry & 0.06 & 97.20 & 0.24 & 0.49 & 0.00 & 1.89 & 0.12 \\
\hline $\mathrm{Sad}$ & 0.43 & 0.43 & 71.90 & 11.01 & 2.07 & 10.83 & 3.35 \\
\hline Afraid & 0.61 & 0.12 & 0.30 & 14.54 & 0.24 & 0.00 & 84.18 \\
\hline Neutral & 0.12 & 0.73 & 2.07 & 0.30 & 95.19 & 0.79 & 0.79 \\
\hline Disgust & 4.99 & 21.53 & 3.22 & 2.98 & 0.06 & 66.48 & 0.73 \\
\hline \multicolumn{8}{|l|}{$\begin{array}{l}\text { Angular-patterned } \\
\text { Mask }\end{array}$} \\
\hline Happy & 97.51 & 0.43 & 0.43 & 0.24 & 0.12 & 1.03 & 0.24 \\
\hline Angry & 0.00 & 97.93 & 0.24 & 0.61 & 0.00 & 1.16 & 0.06 \\
\hline $\mathrm{Sad}$ & 0.36 & 0.67 & 69.65 & 11.92 & 2.43 & 11.74 & 3.22 \\
\hline Afraid & 0.43 & 0.18 & 0.24 & 15.88 & 0.43 & 0.18 & 82.66 \\
\hline Neutral & 0.00 & 0.49 & 1.76 & 0.30 & 95.92 & 0.85 & 0.67 \\
\hline Disgust & 4.99 & 22.81 & 2.25 & 2.49 & 0.18 & 66.61 & 0.67 \\
\hline
\end{tabular}


Given the very high accuracy of emotional recognition even in the masked conditions, we suspected that our participants were learning due to multiple exposure to the same masked faces. To examine this possibility, we conducted a nominal logistic regression with Participant as categorical random independent variable, Order as continuous independent variable, their interaction, and emotion recognition accuracy Score as a categorical response. The results demonstrated a strong and significant Participant effect (Log-likelihood Chi-Square $=1391.84$, $\mathrm{df}=136, p<.0001)$ indicating variability in participants' responses. The effect of Order was moderate and also significant (Chi-Square $=5.43, \mathrm{df}=1, p=.02)$, indicating that, overall, participants learned in the process, however not a lot. The Participant*Order interaction was strong and significant $($ Chi-Square $=216.24, \mathrm{df}=136, p<.0001)$, indicating that participants learned differently - while some improved, some did not change or even worsened their performance.

\section{Pattern, Color and Preference Ratings of Masks without faces}

One-sample t-tests on the ratings of Angularity demonstrated all angular patterns were rated as significantly higher in angularity than chance (test value $=4$ ), $p s<.001$. All round patterns were rated as significantly lower in angularity than chance, $p s<.001$. Black mask angularity ratings did not differ from chance; however, White mask was rated as significantly lower in angularity $(p=.039)$, similar to round patterned masks.

The similar analysis of Color ratings revealed that in all pairs with normal and colorinverted patterns, one was rated as significantly whiter and the other as less white than chance (test value $=4$ ), all $p s<.001$ except for one pattern (Shown in Figure $1-$ Angular, Predominantly White and Sad), consistently judged as predominantly white, but only marginally significantly $(p=.082)$. Black mask was rated as significantly less white than chance $(p<.001)$, 
and White mask was rated as significantly more white than chance $(p<.001)$. White mask was perceived as significantly predominantly white than black mask $(p=.001)$, whereas Angular and Curved patterns did not significantly differ in terms of the perceived color.

Repeated measures ANOVA revealed a significant effect of mask appearance on a preference to mask wearing, $F(563.212,3.902)=144.327, p<.001\left(\eta_{\mathrm{p}}^{2}=.515\right)$. Preference to wear a Black mask $(M=6.10, S D=1.526)$ was the highest compared to other masks (all $p s<$ $.001)$. Preference to wear a White mask $(M=5.38, S D=1.997)$ was higher than for masks with Angular $(M=3.09, S D=1.85)$ and Curved $(M=2.87, S D=1.78)$ patterns (both $p s<.001)$. Preference to wear a mask with Angular patterns only tended to be higher than for Curved ( $p$ $=.085)$.

\section{Emotional recognition and Individual differences self-reports}

We used Pearson's correlation analysis to examine the relationships between emotional recognition and individual differences measures (Table 2). 


\begin{tabular}{|c|c|c|c|c|c|c|c|c|c|c|c|c|c|c|c|c|c|c|}
\hline & FMPS & FMPS1 & FMPS2 & FMPS3 & FMPS4 & FMPS5 & FMPS6 & FMPS7 & FMPS8 & FMPS9 & FMP+ & Outdoor & Indoor & Risks & STAIS & STAIT & EMO-R & EMO-D. \\
\hline ANG ACC & -.143 & $-.179^{*}$ & -.107 & -.122 & .045 & -.119 & -.123 & -.154 & -.097 & -.156 & -.047 & .02 & .104 & -.02 & -.149 & $-.172^{*}$ & -.043 & -.026 \\
\hline CUR ACC & $-.171^{*}$ & $-.205^{*}$ & -.133 & -.13 & .08 & -.116 & -.167 & $-.173^{*}$ & -.161 & $-.170^{*}$ & -.034 & .013 & .086 & .028 & -.126 & $-.170^{*}$ & -.08 & -.046 \\
\hline WHITE ACC & -.161 & $-.201^{*}$ & -.123 & $-.200^{*}$ & .015 & -.079 & -.115 & -.134 & -.159 & $-.229^{* *}$ & -.044 & -.01 & .037 & .064 & -.164 & $-.226^{* *}$ & -.137 & -.076 \\
\hline BLACK ACC & -.113 & -.134 & -.156 & -.016 & .003 & -.073 & -.101 & -.077 & -.1 & -.068 & .047 & .052 & .03 & -.01 & -.034 & -.133 & -.049 & .074 \\
\hline NOMASK ACC & -.024 & -.016 & -.062 & -.099 & .037 & .027 & .01 & -.028 & -.064 & -.028 & .034 & .107 & .102 & .014 & -.147 & -.011 & -.045 & -.08 \\
\hline ANG CONF & $-.174^{*}$ & -.142 & -.113 & $-.191^{*}$ & -.101 & -.14 & -.159 & $-.183^{*}$ & -.065 & -.165 & .106 & .005 & $.194^{*}$ & .083 & -.062 & -.11 & .091 & .128 \\
\hline CUR CONF & $-.177^{*}$ & -.139 & -.134 & $-.192^{*}$ & -.098 & -.136 & -.163 & $-.191^{*}$ & -.061 & $-.175^{*}$ & .112 & -.005 & $.204^{*}$ & .071 & -.071 & -.131 & .082 & .11 \\
\hline WHITE CONF & $-.189^{*}$ & -.129 & -.149 & $-.219^{*}$ & -.081 & -.147 & $-.188^{*}$ & $-.212^{*}$ & -.075 & $-.184^{*}$ & .118 & -.045 & $.217^{*}$ & .111 & -.066 & -.128 & .069 & .112 \\
\hline BLACK CONF & $-.181^{*}$ & -.166 & -.094 & $-.178^{*}$ & -.148 & -.134 & -.164 & $-.178^{*}$ & -.061 & -.16 & .112 & .02 & .163 & .028 & -.056 & -.082 & .104 & .133 \\
\hline NOMASK CONF & .035 & .014 & .031 & -.065 & .03 & .076 & .068 & -.058 & .065 & -.02 & .11 & .058 & .153 & .1 & -.021 & -.018 & .162 & .043 \\
\hline ANG FC & .1 & .019 & .077 & .105 & .081 & .077 & .026 & $.171^{*}$ & .086 & .088 & -.127 & .077 & -.084 & -.005 & -.002 & .051 & .079 & -.014 \\
\hline CUR FC & $.244^{* *}$ & .149 & $.174^{*}$ & $.188^{*}$ & .085 & $.197^{*}$ & $.185^{*}$ & $.310^{* *}$ & $.205^{*}$ & $.266^{* *}$ & -.112 & 0.1 & -.029 & -.049 & .071 & .032 & .025 & -.051 \\
\hline WHITE FC & .131 & .07 & .098 & .167 & .049 & .092 & .157 & $.168^{*}$ & .036 & .132 & -.151 & .103 & -.084 & -.075 & -.059 & -.066 & -.117 & -.087 \\
\hline BLACK FC & .069 & .075 & .08 & .112 & .081 & .038 & .019 & .06 & .002 & .14 & -.04 & .049 & $-.181^{*}$ & .004 & -.14 & -.083 & -.022 & $-.169^{*}$ \\
\hline NOMASK FC & -.076 & -.073 & -.066 & -.002 & -.007 & -.072 & -.067 & -.051 & -.09 & -.02 & -.017 & -.055 & -.116 & .105 & $-.191^{*}$ & -.114 & -.155 & -.001 \\
\hline ANG PS & $.226^{* *}$ & .112 & $.236^{* *}$ & .166 & .085 & $.226^{* *}$ & .118 & $.288^{* *}$ & $.180^{*}$ & $.206^{*}$ & -.153 & .108 & -.128 & -.063 & .056 & .084 & .11 & -.015 \\
\hline CUR PS & $.293^{* *}$ & $.180^{*}$ & $.256^{* *}$ & $.212^{*}$ & .06 & $.257^{* *}$ & $.206^{*}$ & $.390^{* *}$ & $.233^{* *}$ & $.295^{* *}$ & -.113 & .114 & -.034 & -.065 & .141 & .074 & .033 & -.03 \\
\hline WHITE PS & .166 & .079 & .167 & $.212^{*}$ & .056 & .13 & .15 & $.215^{*}$ & .069 & .141 & $-.189^{*}$ & .098 & -.123 & -.143 & .007 & -.051 & -.071 & -.084 \\
\hline BLACK PS & .146 & .124 & $.185^{*}$ & .149 & .09 & .14 & .062 & .134 & .058 & $.197^{*}$ & -.113 & .089 & $-.178^{*}$ & -.059 & -.095 & -.08 & .021 & $-.169^{*}$ \\
\hline NOMASK PS & .017 & .011 & .05 & .023 & -.079 & .032 & -.006 & .079 & .003 & .023 & .001 & .035 & -.077 & .035 & -.09 & -.048 & -.003 & .017 \\
\hline WHITE PREF & -.059 & -.079 & -.111 & 0.01 & -.034 & -.076 & -.105 & -.027 & .072 & -.119 & .039 & -.025 & .028 & -.009 & .036 & -.073 & -.026 & .046 \\
\hline BLACK PREF & $-.183^{*}$ & -.123 & -.153 & $-.169^{*}$ & -.002 & $-.291^{* *}$ & $-.226^{* *}$ & -.119 & -.043 & $-.169^{*}$ & $.201 *$ & .068 & .108 & -.086 & .043 & .13 & .131 & .06 \\
\hline ANG PREF & $.185^{*}$ & .02 & .134 & .114 & .011 & $.196^{*}$ & $.177^{*}$ & $.264^{* *}$ & $.204^{*}$ & .148 & -.023 & -.006 & $-.271^{* *}$ & $.169^{*}$ & .096 & .054 & .008 & -.046 \\
\hline CUR PREF & $.234^{* *}$ & .096 & $.175^{*}$ & .13 & .014 & $.218^{*}$ & $.221^{* *}$ & $.295^{* *}$ & $.249^{* *}$ & .158 & -.003 & -.029 & $-.255^{* *}$ & .156 & $.200^{*}$ & .086 & .026 & .029 \\
\hline
\end{tabular}

Table 1. Correlations between Emotional recognition and Individual differences self-reports. Note: ** Correlation is significant at the 0.01 level (2-tailed). * Correlation is significant at the 0.05 level (2-tailed). ${ }^{+}$Correlation is significant at the 0.1 level (2-tailed).

FMPS scales: 1-Comfort, 2- Efficacy doubt, 3- Access, 4- Compensation, 5- Inconvenience, 6- Appearance, 7- Attention, 8-

Independence, 9- Emotion (new). ANG-Angular, CUR-Curved, ACC-Accuracy, CONF- Confidence, FC and PS- time to first click and page submit, PREF - Preference. 
Notably, scores distribution analysis (FMPS: $M=2.77, S D=1.14$; MPS+: $M=22.57, S D$ $=1.13$; Risks Estimate: $M=2.56, S D=1.67$; Mask Wearing Outdoors: $M=6.55, S D=1.05$; Mask Wearing Indoors: $M=5.92, S D=1.73$ ) indicates that, overall, our Turkish student sample had rather positive attitudes towards masks and high wearing habits. In fact, another study conducted with 3040 Turkish university students in April 2020 reported use of masks among university students was 50\%, much higher than expected as there was no legal obligation to do so in the beginning of the pandemic.

The findings indicate that, overall, negative attitudes towards masks (higher scores on FMPS) tended to be negatively related to accuracy in emotional recognition only for masked but not unmasked faces. At the same time, FMPS positively correlated with the time of emotion recognition in faces with curved and angular masks. Consistent trends were observed for separate FMPS scales (see Table 2). FMPS negatively correlated with frequency of wearing masks indoors $(r=-.214, p=.013)$. Wearing masks indoors positively correlated with Confidence in emotional recognition.

Interestingly, preferences to wear Black masks (that were the most prevalent as shown in the previous analysis) negatively correlated with FMPS, but preferences to wear the least preferred (patterned) masks positively correlated with FMPS. At the same time preferences to wear patterned masks were negatively related to actual wearing masks indoors and perceived risks of COVID. FMP+ (assessing positive attitudes towards wearing masks) positively correlated with preference for Black mask wearing. Preferences to wear White masks were positively correlated with higher accuracy ( $r$ s ranged between .247 and $.313 ; p$ s $\leq .004)$ and confidence ( $r$ ranged between .256 and $.293 ; p s \leq .003)$ in recognition of all kinds of masked but not unmasked faces. Those who preferred Black masks also had higher confidence $(r \mathrm{~s}$ 
ranged between .200 and $.210 ; p s \leq .019)$ in recognition of all kinds of masked but not unmasked faces.

Consistent with our expectations, higher anxiety, especially trait anxiety, was associated with lower accuracy in recognizing emotions on masked faces ( $r$ s ranged between - 170 and $.226 ; p s \leq .047$, except Black). State and trait anxiety were positively associated with several scales of FMPS and at the same time with FMP+ $(r=.237, p=.005)$. Emotional Range correlated with both state $(r=.210, p=.014)$ and trait $(r=.261, p=.002)$ anxiety, and also with FMPS comfort scale $(r=.206, p=.016)$.

\section{Sex differences}

Males and females did not significantly differ in Emotional Recognition of either Masked or Unmasked faces in terms of Accuracy, Confidence and First Click Response Time, but males were faster in Page Submit RT when recognizing Unmasked faces, [ $t$ (114.74) = -2.580, p=.011; males: $M=6.13, S D=1.65$, females: $M=6.99, S D=2.18)]$. There were no sex differences in mask preferences (both sexes preferred black masks).

Independent-samples t-test revealed significant sex differences, favoring females, in Emotional Differentiation $[t(135)=-3.648, p<.001$; males: $M=3.68, S D=.58$, females: $M=4.09$, $S D=.63)]$ and in Emotional Range $[t(135)=-2.730, p=.007$; males: $M=3.64, S D=.61$, females: $M=3.98, S D=.71)]$, and no other significant differences in other self-report measures.

\section{General Discussion}

Our study explored the effects of face masks' designs on emotional recognition. We examined emotional recognition in faces uncovered and covered by masks of different patterns (curved vs. angular) and colors (white and black). Consistent with literature (Carbon, 2020; 
Freud et al., 2020; Marini et al., 2021) and our H1, we found that the presence of masks impaired emotional recognition. Overall, accuracy and confidence dropped, and reaction time increased for masked faces compared to unmasked faces. The appearance of the mask in terms of pattern and color seem not to play a significant role in overall accuracy, confidence and speed of emotional recognition. Contrary to our expectations, we did not find main effects of angularity or color on emotion recognition. Faces covered by masks with different patterns and amount of white or black colors were recognized approximately at the same rate. This may be the case that mask design does not matter for the emotional recognition of masked faces, but it is also possible that we did not detect the effect for a number of reasons. Note, that we utilized rather complex patterns on masks (e.g., zigzags and wavy). Though our participants perceived these shapes as intended (e.g., zigzag as angular and wavy as curvy), simpler and more obvious designs (e.g., one big circle, one triangle) could have made the perception of angular vs. curvy more salient. Subsequent studies should investigate the effects of masks with simpler designs. In addition, the size and the density of the pattern may matter.

Notably, the found difference between masked and unmasked conditions was not very large. Unlike previous research, we found a surprisingly high emotion recognition rate (ceiling effect - especially for happy, angry, and neutral emotions). One reason could be using a scientific database with models clearly posing the emotions. Carbon (2020), who used the same database, also reported very high performance in recognition of unmasked faces (though he did not find significant differences between masked and unmasked face recognition for fearful and neutral emotional states). However, Carbon did not find such a high rate in recognition of masked faces. Notably, unlike Carbon, we used only the faces of young models. Carbon found that performance in recognizing emotions of elderly faces was lower than for middle-aged or 
young faces. Research suggested that young adults are better at recognizing emotions compared to elderly (Grundmann et al., 2021). Since our participants were university students, the fact that the age of the models matched the age of the participants could also have made the task easier. In addition, our participants were students from a Turkish university. Previous studies showed that there may be differences across cultures in the way individuals read emotions. Some cultures (e.g., Eastern) were found to focus on eyes when reading emotions (Jack et al., 2009). Perhaps, the style of reading expressions might have facilitated emotion recognition in masked faces. Future research, therefore, should explore the proposed relationships employing other samples (various cultures as well as a wider range of ages) and broader stimuli (age-wise as well as trained vs. untrained models). Another reason for high performance can be repeated exposure to the same faces that could have increased the recognition of emotions. Notably, participants did not see unmasked faces before completing masked faces' emotional recognition task, so high performance cannot be simply due to the recognition of previously seen unmasked faces. Yet, we revealed a small learning effect - an increase in emotional recognition accuracy depending on the order of a trial. Our results indicate that people may improve the accuracy of masked faces' emotional identification just from the repeated exposure within a short-term timeframe (our task took less than $20 \mathrm{~min}$ ). Similarly, previous studies showed that individual differences in mask exposure influenced the use of visual cues from the face. A longitudinal study (two controlled experiments with 6 months apart) assessed the perceived emotional similarity between a pair of unmasked faces (Barrick et al., 2020). As mask exposure increases, there is an increase in the eye cue use. This literature indicates that face perception is malleable and longer exposure to masked faces may actually change the way we read emotions. The longer people interact with others that wear masks, the more they learn to focus on visual cues from the eye area of the face. Whereas 
there is some proof that during pandemics people may have learned to recognize masked faces better with time. which may explain better performance in recognition.

Furthermore, in support of $\mathrm{H} 2$, we found significant differences between the recognition of different emotions for masked vs. unmasked faces. We found that the presence of masks mostly impaired Afraid, Sad, and Disgust emotions. We found that masks impaired the recognition of some emotions that involve more of the lower part of the face (e.g., disgust) but did not find for the others (Happy was the least affected, inconsistent with some other studies (Carbon, 2020), possibly because the model had a Duchenne smile). Confidence ratings showed more clear differences between masked and unmasked faces' than recognition performance (for example, for Happy and Neutral emotions). At the same time, there were less differences in confidence ratings between the emotions. While recognition accuracy for Afraid, Sad and Disgust was relatively low, the confidence in recognition of this emotional state was comparable with other emotions. Furthermore, we did not find that angular and darker designs lead to more negative attributions of emotions whereas curvy and lighter patterns yield more positive emotion perceptions. The emotions were confused more frequently in masked faces, but we did not observe any negative or positive biases depending on the mask design. Overall, these results indicate that mask design did not significantly contribute to specific misinterpretations of emotions in masked faces. Our findings suggest that people may be quite successful in ignoring irrelevant visual information while focusing on reading emotions from eyes only.

Finally, we expected to find the relationship between individual difference measures and emotional recognition $(\mathrm{H} 3)$. As predicted, attitudes towards masks were related to performance in reading emotions. People with negative attitudes towards masks appeared to be less accurate and less confident in recognition of emotions on masked faces. We cannot tell, though, whether 
negative attitudes lead to worse performance or vice versa. Notably, this relationship did not exist for unmasked faces, so negative attitudes towards masks were not related to emotional recognition skills in general. People frequently wearing masks indoors were more confident in emotional recognition, again, only for masked faces. Thus, the attitudes and mask-wearing behavior were specifically related to sensitivity to emotions on masked but not unmasked faces.

Our research suggests that certain individual traits and attitudes may predict the ability to recognize emotions on masked but not unmasked faces. It is possible that the selective impairment in processing masked emotions may be related to certain individual differences. As some literature suggests, reading emotions from isolated face regions may be specifically impaired in some populations (e.g., autistic - Baron-Cohen et al. 1997b; Pazhoohi et al., 2021; Ramachandra \& Longacre, 2022). Possibly, occluding mouth regions may be disadvantageous for individuals with specific traits. Future studies should explore how people with superior ability for recognizing faces (super recognizers; Russell \& Nakayama, 2009; Noyes, et al. 2017) are affected by masks. Consistent with our expectations, higher anxiety was associated with lower emotional recognition accuracy in masked faces as well as with negative attitudes towards masks. At the same time, anxiety was positively associated with FMP+ (positive attitudes towards masks). Thus, both positive and negative attitudes may be potentially motivated by anxiety. Overall, our findings demonstrate the existence of the relationship between individual differences in emotional processing, attitudes towards masks, and reading emotions from masked faces, though it may be rather complex. Future studies need to further search for the individual differences that predict emotional processing in masked faces.

We observed some consistent relationships among preferences for specific mask appearances, attitudes towards masks, and emotional recognition. The majority of participants 
demonstrated positive attitudes towards mask wearing and preferred non-patterned black and white masks. Those who preferred white unpatterned masks had higher accuracy and confidence in recognition of all kinds of masked but not unmasked faces. Similarly, those who preferred black (also unpatterned) masks had higher confidence in recognition of all kinds of masked but not unmasked faces. Interestingly, those with negative attitudes towards masks preferred patterned masks. So it may be that they perceive masks as entertaining, funny or a fashion accessory rather than a protective medical item. At the same time, people frequently wearing masks indoors (which is more important than wearing masks outdoors in the context of COVID19) did not prefer angular and curved masks, which may indicate that they did not perceive patterned masks seriously. Interestingly, those with negative attitudes towards masks also tended to be slower in making decisions about emotional states especially when the patterned masks were worn. Possibly, because they perceived patterned masks more as a clothing item rather than medical device (medical masks are non-patterned masks) and therefore took more time to aesthetically examine them.

COVID-19 pandemic has resulted in multiple paradigm shifts in society (Howe et al., 2020), creating habits such as wearing masks that are likely to stay even after the pandemic subsides (Sheth, 2020). Such behavior patterns are likely to have long-term effects on how people perceive socialization and engage in social interactions. More studies, therefore, are needed to examine how both mask appearance and attitudes towards masks may affect emotional recognition as well as how they may interact. 


\section{Declaration}

\section{Ethics Approval and consent to participate}

The study was approved by Sabanci University ethics committee (Protocol No: FASS-2019-62) and all the participants received a consent form and approved it.

\section{Consent for publication}

NA

\section{Availability of Data and Materials}

The datasets generated and/or analyzed during the current study will be available in the OSF repository, upon the acceptance of the manuscript.

\section{Funding}

The authors have individual research funds provided by Sabanci University.

\section{Authors Contributions}

$\mathrm{OB}$ and KDD were involved in Conceptualization, Methodology, and Project administration. $\mathrm{OB}$ analyzed the data. OB and KDD interpreted the analyses. OB and KDD did the literature review and write-up. All authors read and approved the final manuscript.

\section{Competing interests}

The authors declare that they have no competing interests

\section{Acknowledgements}

We would like to thank Lev Yampolsky (East Tennessee State University) who provided insight and expertise in statistical analysis of order effects. 


\section{References}

Argyle, M. (1970). Eye-contact and distance: A reply to Stephenson and Rutter. British Journal of Psychology , 61(3), 395-396. https://doi.org/10.1111/j.2044-8295.1970.tb01258.x

Aronoff, J., Barclay, A. M., \& Stevenson, L. A. (1988). The recognition of threatening facial stimuli. Journal of Personality and Social Psychology, 54(4), 647-655. https://doi.org/10.1037//0022-3514.54.4.647

Aronoff, J., Woike, B. A., \& Hyman, L. M. (1992). Which are the stimuli in facial displays of anger and happiness? Configurational bases of emotion recognition. Journal of Personality and Social Psychology, 62(6), 1050-1066. https://doi.org/10.1037/0022-3514.62.6.1050

Bani, M., Russo, S., Ardenghi, S., Rampoldi, G., Wickline, V., Nowicki, S., Jr, \& Strepparava, M. G. (2021). Behind the Mask: Emotion Recognition in Healthcare Students. Medical Science Educator, 1-5. https://doi.org/10.1007/s40670-021-01317-8

Bar, M., \& Neta, M. (2006). Humans prefer curved visual objects. Psychological Science, 17(8), 645-648. https://doi.org/10.1111/j.1467-9280.2006.01759.x

Baron-Cohen, S., Jolliffe, T., Mortimore, C., \& Robertson, M. (1997a). Another advanced test of theory of mind: Evidence from very high functioning adults with autism or Asperger syndrome. Journal of Child psychology and Psychiatry, 38(7), 813-822. https://doi.org/10.1111/j.1469-7610.1997.tb01599.x

Baron-Cohen, S., Wheelwright, S., \& Jolliffe, A. T. (1997b). Is there a "language of the eyes"? Evidence from normal adults, and adults with autism or Asperger syndrome. Visual Cognition, 4(3), 311-331. https://doi.org/10.1080/713756761

Barrick, E., Thornton, M. A., \& Tamir, D. (2020). Mask exposure during COVID-19 changes emotional face processing. https://psyarxiv.com/yjfg3/download?format=pdf 
Bassili, J. N. (1979). Emotion recognition: The role of facial movement and the relative importance of upper and lower areas of the face. Journal of Personality and Social Psychology, 37(11), 2049-2058. https://doi.org/10.1037/0022-3514.37.11.2049

Beaudry, O., Roy-Charland, A., Perron, M., Cormier, I., \& Tapp, R. (2014). Featural processing in recognition of emotional facial expressions. Cognition \& Emotion, 28(3), 416-432. https://doi.org/10.1080/02699931.2013.833500

Becker, L., van Rompay, T. J. L., Schifferstein, H. N. J., \& Galetzka, M. (2011). Tough package, strong taste: The influence of packaging design on taste impressions and product evaluations. Food Quality and Preference, 22(1), 17-23. https://doi.org/10.1016/j.foodqual.2010.06.007

Biermann, M., Schulze, A., Unterseher, F., Atanasova, K., Watermann, P., Krause-Utz, A., Stahlberg, D., Bohus, M., \& Lis, S. (2021). Trustworthiness appraisals of faces wearing a surgical mask during the Covid-19 pandemic in Germany: An experimental study. PloS One, 16(5), e0251393. https://doi.org/10.1371/journal.pone.0251393

Blais, C., Fiset, D., Roy, C., Saumure Régimbald, C., \& Gosselin, F. (2017). Eye fixation patterns for categorizing static and dynamic facial expressions. Emotion , 17(7), 1107-1119. https://doi.org/10.1037/emo0000283

Blazhenkova, O. (2017). Boundary Extension in Face Processing. I-Perception, 8(5), 2041669517724808. https://doi.org/10.1177/2041669517724808

Blazhenkova, O., \& Dogerlioglu-Demir, K. (2020). The shape of the pill: Perceived effects, evoked bodily sensations and emotions. PloS One, 15(9), e0238378. https://doi.org/10.1371/journal.pone.0238378

Blazhenkova, O., \& Kumar, M. M. (2018). Angular Versus Curved Shapes: Correspondences 
and Emotional Processing. Perception, 47(1), 67-89. https://doi.org/10.1177/0301006617731048

Bombari, D., Schmid, P. C., Schmid Mast, M., Birri, S., Mast, F. W., \& Lobmaier, J. S. (2013). Emotion recognition: the role of featural and configural face information. Quarterly Journal of Experimental Psychology, 66(12), 2426-2442. https://doi.org/10.1080/17470218.2013.789065

Bruce, V., \& Young, A. (1986). Understanding face recognition. British Journal of Psychology, 77 ( Pt 3), 305-327. https://doi.org/10.1111/j.2044-8295.1986.tb02199.x

Calvo, M. G., Fernández-Martín, A., \& Nummenmaa, L. (2014). Facial expression recognition in peripheral versus central vision: role of the eyes and the mouth. Psychological Research, 78(2), 180-195. https://doi.org/10.1007/s00426-013-0492-x

Carbon, C.-C. (2010). The cycle of preference: long-term dynamics of aesthetic appreciation. Acta Psychologica, 134(2), 233-244. https://doi.org/10.1016/j.actpsy.2010.02.004

Carbon, C.-C. (2020). Wearing Face Masks Strongly Confuses Counterparts in Reading Emotions. Frontiers in Psychology, 11, 566886. https://doi.org/10.3389/fpsyg.2020.566886

Carbon, C.-C., \& Serrano, M. (2021). The Impact of Face Masks on the Emotional Reading Abilities of Children-A Lesson From a Joint School-University Project. I-Perception, 12(4), 20416695211038265. https://doi.org/10.1177/20416695211038265

Cimbalo, R. S., Beck, K. L., \& Sendziak, D. S. (1978). Emotionally Toned Pictures and Color Selection for Children and College Students. The Journal of Genetic Psychology, 133(2), 303-304. https://doi.org/10.1080/00221325.1978.10533389

Clarke, T., \& Costall, A. (2008). The emotional connotations of color: A qualitative investigation. Color Research and Application, 33(5), 406-410. 
https://doi.org/10.1002/col.20435

Corpuz, J. C. G. (2021). Adapting to the culture of "new normal": an emerging response to COVID-19. Journal of Public Health , 43(2), e344-e345.

https://doi.org/10.1093/pubmed/fdab057

De Bortoli, M., \& Maroto, J. (2001). Colours across cultures: Translating colours in interactive marketing communications. globalpropaganda.fresa.net. http://www.globalpropaganda.fresa.net/articles/TranslatingColours.pdf

Deighton, R. M., \& Traue, H. C. (2007). Emotion recognition patterns in patients with panic disorder. Depression and anxiety, 24(3), 223-226.

Demenescu, L. R., Kortekaas, R., den Boer, J. A., \& Aleman, A. (2010). Impaired attribution of emotion to facial expressions in anxiety and major depression. PloS one, 5(12), e15058.

Duchaine B, Nakayama K. Super-recognizers: people with extraordinary face recognition ability. Psychonomic bulletin \& review. 2009;16(2):252-7. pmid:19293090

Ebner, N. C., Riediger, M., \& Lindenberger, U. (2010). FACES-A database of facial expressions in young, middle-aged, and older women and men: Development and validation. Behavior Research Methods, 42(1), 351-362. https://doi.org/10.3758/BRM.42.1.351

Eisenbarth, H., \& Alpers, G. W. (2011). Happy mouth and sad eyes: scanning emotional facial expressions. Emotion , 11(4), 860-865. https://doi.org/10.1037/a0022758

Ekman, P., Friesen, W. V., \& Ellsworth, P. (1972). Emotion in the Human Face: Guide-lines for Research and an Integration of Findings: Guidelines for Research and an Integration of Findings. Pergamon.

Fischer, A. H., Gillebaart, M., Rotteveel, M., Becker, D., \& Vliek, M. (2012). Veiled Emotions: 
The Effect of Covered Faces on Emotion Perception and Attitudes. Social Psychological and Personality Science, 3(3), 266-273. https://doi.org/10.1177/1948550611418534

Fisher, K., Towler, J., \& Eimer, M. (2016). Effects of contrast inversion on face perception depend on gaze location: Evidence from the N170 component. Cognitive Neuroscience, 7(1-4), 128-137. https://doi.org/10.1080/17588928.2015.1053441

Freud, E., Stajduhar, A., Rosenbaum, R. S., Avidan, G., \& Ganel, T. (2020). The COVID-19 pandemic masks the way people perceive faces. Scientific Reports, 10(1), 1-8. https://doi.org/10.1038/s41598-020-78986-9

Funk, D., \& Nelson, O. N. (2006). Colour and product choice: a study of gender roles. Management Research News, 29(1/2), 41-52. https://doi.org/10.1108/01409170610645439

Genç, Ç., Colley, A., Löchtefeld, M., \& Häkkilä, J. (2020). Face mask design to mitigate facial expression occlusion. Proceedings of the 2020 International Symposium on Wearable Computers, 40-44. https://doi.org/10.1145/3410531.3414303

Gilad, S., Meng, M., \& Sinha, P. (2009). Role of ordinal contrast relationships in face encoding. Proceedings of the National Academy of Sciences of the United States of America, 106(13), 5353-5358. https://doi.org/10.1073/pnas.0812396106

Goldstein, A. G., \& Mackenberg, E. J. (1966). Recognition of human faces from isolated facial features: A developmental study. Psychonomic Science, 6(4), 149-150. https://doi.org/10.3758/BF03328001

Gómez-Puerto, G., Rosselló, J., Corradi, G., Acedo-Carmona, C., Munar, E., \& Nadal, M. (2018). Preference for curved contours across cultures. Psychology of Aesthetics, Creativity, and the Arts, 12(4), 432. https://psycnet.apa.org/journals/aca/12/4/432/

Grundmann, F., Epstude, K., \& Scheibe, S. (2021). Face masks reduce emotion-recognition 
accuracy and perceived closeness. PloS One, 16(4), e0249792.

https://doi.org/10.1371/journal.pone.0249792

Hanada, M. (2018). Correspondence analysis of color-emotion associations. Color Research and Application, 43(2), 224-237. https://doi.org/10.1002/col.22171

Hevner, K. (1935). Experimental studies of the affective value of colors and lines. The Journal of Applied Psychology, 19(4), 385-398. https://doi.org/10.1037/h0055538

Howard, M. C. (2020). Understanding face mask use to prevent coronavirus and other illnesses: Development of a multidimensional face mask perceptions scale. British Journal of Health Psychology, 25(4), 912-924. https://doi.org/10.1111/bjhp.12453

Howe, D. C., Chauhan, R. S., Soderberg, A. T., \& Buckley, M. R. (2020). Paradigm shifts caused by the COVID-19 pandemic. Organizational Dynamics, https://doi.org/10.1016/j.orgdyn.2020.100804.

Hussain, M. Z. (1972). Effect of shape of medication in treatment of anxiety states. The British Journal of Psychiatry: The Journal of Mental Science, 120(558), 507-509. https://doi.org/10.1192/bjp.120.558.507

Itier, R. J., Alain, C., Sedore, K., \& McIntosh, A. R. (2007). Early face processing specificity: it's in the eyes! Journal of Cognitive Neuroscience, 19(11), 1815-1826. https://doi.org/10.1162/jocn.2007.19.11.1815

Jack, R. E., Blais, C., Scheepers, C., Schyns, P. G., \& Caldara, R. (2009). Cultural confusions show that facial expressions are not universal. Current Biology: CB, 19(18), 1543-1548. https://doi.org/10.1016/j.cub.2009.07.051

James, T. W., Huh, E., \& Kim, S. (2010). Temporal and spatial integration of face, object, and scene features in occipito-temporal cortex. Brain and Cognition, 74(2), 112-122. 
https://doi.org/10.1016/j.bandc.2010.07.007

Janik, S. W., Wellens, A. R., Goldberg, M. L., \& Dell'Osso, L. F. (1978). Eyes as the center of focus in the visual examination of human faces. Perceptual and Motor Skills, 47(3 Pt 1), 857-858. https://doi.org/10.2466/pms.1978.47.3.857

Jiang, Y., Gorn, G. J., Galli, M., \& Chattopadhyay, A. (2015). Does Your Company Have the Right Logo? How and Why Circular- and Angular-Logo Shapes Influence Brand Attribute Judgments. The Journal of Consumer Research, 42(5), 709-726. https://doi.org/10.1093/jcr/ucv049

Kang, S.-M., \& Shaver, P. R. (2004). Individual differences in emotional complexity: their psychological implications. Journal of Personality, 72(4), 687-726.

https://doi.org/10.1111/j.0022-3506.2004.00277.x

Labrecque, L. I., \& Milne, G. R. (2012). Exciting red and competent blue: the importance of color in marketing. Journal of the Academy of Marketing Science, 40(5), 711-727. https://doi.org/10.1007/s11747-010-0245-y

Lundholm, H. (1921). The Affective Tone of Lines: Experimental Researches. Psychological Review, 28(1), 43. https://psycnet.apa.org/journals/rev/28/1/43/

Madden, T. J., Hewett, K., \& Roth, M. S. (2000). Managing Images in Different Cultures: A Cross-National Study of Color Meanings and Preferences. Journal of International Marketing, 8(4), 90-107. https://doi.org/10.1509/jimk.8.4.90.19795

Marini, M., Ansani, A., Paglieri, F., Caruana, F., \& Viola, M. (2021). The impact of facemasks on emotion recognition, trust attribution and re-identification. Scientific Reports, 11(1), 5577. https://doi.org/10.1038/s41598-021-84806-5

McCrackin, S., Capozzi, F., Mendell, E., Provencher, S., Mayrand, F., \& Ristic, J. (2021). 
Recognition of emotions is affected by face masks. Journal of Vision, 21(9), 2153-2153. https://doi.org/10.1167/jov.21.9.2153

McKelvie, S. J. (1995). Emotional expression in upside-down faces: Evidence for configurational and componential processing. The British Journal of Social Psychology / the British Psychological Society, 34(3), 325-334. https://doi.org/10.1111/j.20448309.1995.tb01067.x

Noyes, E., Phillips, P. J., \& O’Toole, A. J. (2017). What is a super-recogniser?. In Face processing: Systems, disorders and cultural differences (pp. 173-201). Nova Science Publishers Inc.

Okazaki, S., Yamanami, H., Nakagawa, F., Takuwa, N., \& Kawabata, K. J. D. (2021). Eyes compensate smile when wearing mask. In Research Square. Research Square. https://doi.org/10.21203/rs.3.rs-576144/v1

Palumbo, L., Ruta, N., \& Bertamini, M. (2015). Comparing Angular and Curved Shapes in Terms of Implicit Associations and Approach/Avoidance Responses. PloS One, 10(10), e0140043. https://doi.org/10.1371/journal.pone.0140043

Papanek, V. J. (1995). Green imperative. Thames and Hudson. https://agris.fao.org/agrissearch/search.do?recordID=US201300300688

Pazhoohi, F., Forby, L., \& Kingstone, A. (2021). Facial masks affect emotion recognition in the general population and individuals with autistic traits. PloS One, 16(9), e0257740. https://doi.org/10.1371/journal.pone.0257740

Pellicano, E., Rhodes, G., \& Peters, M. (2006). Are preschoolers sensitive to configural information in faces? Developmental Science, 9(3), 270-277. https://doi.org/10.1111/j.1467-7687.2006.00489.x 
Poffenberger, A. T., \& Barrows, B. E. (1924). The Feeling Value of Lines. The Journal of Applied Psychology, 8(2), 187. https://psycnet.apa.org/journals/apl/8/2/187/

Prkachin, G. C. (2003). The effects of orientation on detection and identification of facial expressions of emotion. British Journal of Psychology , 94(Pt 1), 45-62. https://doi.org/10.1348/000712603762842093

Ramachandra, V., \& Longacre, H. (2022). Unmasking the psychology of recognizing emotions of people wearing masks: The role of empathizing, systemizing, and autistic traits. Personality and Individual Differences, 185, 111249. https://doi.org/10.1016/j.paid.2021.111249

Roberson, D., Kikutani, M., Döge, P., Whitaker, L., \& Majid, A. (2012). Shades of emotion: what the addition of sunglasses or masks to faces reveals about the development of facial expression processing. Cognition, 125(2), 195-206. https://doi.org/10.1016/j.cognition.2012.06.018

Russell, R., Duchaine, B., \& Nakayama, K. (2009). Super-recognizers: People with extraordinary face recognition ability. Psychonomic Bulletin \& Review, 16(2), 252-257.

Saito, M. (1996). Comparative studies on color preference in Japan and other Asian regions, with special emphasis on the preference for white. Color Research and Application, 21(1), 3549. https://doi.org/10.1002/(sici)1520-6378(199602)21:1<35::aid-col4>3.0.co;2-6

Saint, S. A., \& Moscovitch, D. A. (2021). Effects of mask-wearing on social anxiety: an exploratory review. Anxiety, Stress, \& Coping, 1-16.

Sheldon, K. M., Goffredi, R., \& Corcoran, M. (2021). The Glow Still Shows: Effects of Facial Masking on Perceptions of Duchenne Versus Social Smiles. Perception, 50(8), 720-727. https://doi.org/10.1177/03010066211027052 
Sheth, J. (2020). Impact of Covid-19 on consumer behavior: Will the old habits return or die? Journal of Business Research, 117, 280-283.

Silchenko, K., \& Visconti, L. M. (2021). Facemask: from pandemic to marketplace iconicity. Consumption Markets \& Culture, 1-24. https://doi.org/10.1080/10253866.2021.1909004

Sliburyte, L., \& Skeryte, I. (2014). What we know about consumers' color perception. Procedia, Social and Behavioral Sciences, 156, 468-472. https://doi.org/10.1016/j.sbspro.2014.11.223

Smith, M. L., Cottrell, G. W., Gosselin, F., \& Schyns, P. G. (2005). Transmitting and decoding facial expressions. Psychological Science, 16(3), 184-189. https://doi.org/10.1111/j.09567976.2005.00801.x

Surcinelli, P., Codispoti, M., Montebarocci, O., Rossi, N., \& Baldaro, B. (2006). Facial emotion recognition in trait anxiety. Journal of anxiety disorders, 20(1), 110-117. https://doi.org/10.1016/j.janxdis.2004.11.010

Taylor, S., \& Asmundson, G. J. G. (2021). Negative attitudes about facemasks during the COVID-19 pandemic: The dual importance of perceived ineffectiveness and psychological reactance. PloS One, 16(2), e0246317. https://doi.org/10.1371/journal.pone.0246317

Wegrzyn, M., Vogt, M., Kireclioglu, B., Schneider, J., \& Kissler, J. (2017). Mapping the emotional face. How individual face parts contribute to successful emotion recognition. PloS One, 12(5), e0177239. https://doi.org/10.1371/journal.pone.0177239

Willis, J., \& Todorov, A. (2006). First impressions: making up your mind after a 100-ms exposure to a face. Psychological Science, 17(7), 592-598. https://doi.org/10.1111/j.14679280.2006.01750.x

Zhang, Y., Feick, L., \& Price, L. J. (2006). The impact of self-construal on aesthetic preference for angular versus rounded shapes. Personality \& Social Psychology Bulletin, 32(6), 794- 
805. https://doi.org/10.1177/0146167206286626

Zsido, A. N., Teleki, S. A., Csokasi, K., Rozsa, S., \& Bandi, S. A. (2020). Development of the short version of the spielberger state - trait anxiety inventory. Psychiatry Research, 291, 113223. https://doi.org/10.1016/j.psychres.2020.113223 